LAWRENCE LIVERMORE NATIONAL LABORATORY

\title{
Sub-picosecond Streak Camera Measurements at LLNL: From IR to x-rays Title
}

Jaroslav Kuba*, Ronnie Shepherd, Rex Booth, Rick Stewart, Edward C. W. Lee, Patrick Audebert, John K. Crane, Robert. R. Cross, Paul T. Springer

\section{December 19, 2003}

Proceedings of a talk submitted to the Ultrafast X-ray detectors and applications, Section Ultrafast Detector Applications, Zenghu Chang, Jean-Claude Kieffer, Jerome B. Hastings, conference Chairs, SPIE International Symposium on Optical Science and Technology, San Diego, California, USA, 3 - 8 August 2003 


\title{
Sub-picosecond Streak Camera Measurements at LLNL: From IR to X-rays
}

\author{
Jaroslav Kuba ", Ronnie Shepherd, Rex Booth, Rick Stewart, \\ Edward C. W. Lee, Patrick Audebert, John K. Crane, Robert. R. Cross, \\ Paul T. Springer \\ Lawrence Livermore National Laboratory, Livermore, CA, USA
}

\begin{abstract}
An ultra fast, sub-picosecond resolution streak camera has been recently developed at the LLNL. The camera is a versatile instrument with a wide operating wavelength range. The temporal resolution of up to $300 \mathrm{fs}$ can be achieved, with routine operation at $500 \mathrm{fs}$. The streak camera has been operated in a wide wavelength range from IR to $\mathrm{x}$-rays up to $2 \mathrm{keV}$. In this paper we briefly review the main design features that result in the unique properties of the streak camera and present its several scientific applications: (1) Streak camera characterization using a Michelson interferometer in visible range, (2) temporally resolved study of a transient x-ray laser at $14.7 \mathrm{~nm}$, which enabled us to vary the $\mathrm{x}$-ray laser pulse duration from $\sim 2-6 \mathrm{ps}$ by changing the pump laser parameters, and (3) an example of a time-resolved spectroscopy experiment with the streak camera.
\end{abstract}

\section{INTRODUCTION}

The advent of the Chirped-Pulse-Amplification technique in high-power lasers has enabled the generation of ultra-short (sub-picosecond) laser pulses opening a range of new experimental areas in ultra-fast physics, e. g. relativistic laser generated plasmas, transient $\mathrm{x}$-ray laser schemes, $\mathrm{K}-\alpha$ source development and many others. The new ultra-fast physics often requires a diagnostic capability that exceeds most current technologies. Thus there is great interest in developing new ultra-fast diagnostics to study dynamics of these processes in the new regime.

In 1990 the 2-ps resolution barrier was successfully broken in x-ray streak camera design [1]. Although these streak cameras provided an excellent service in understanding the processes involved in laser-plasma interactions, it was clear that further developments were required to study the ultra-short phenomena on a sub-picosecond timescale. Despite a large need and research effort by several groups, the x-ray tube design did not show a significant improvement for almost 10 years.

To address the needs in the field, the Lawrence Livermore National Laboratory research effort focused on these goals:

- Maximization of the streak camera's resolution into a sub-picosecond level

- Development of a compact design, and

- Development of a design that would enable us to carry out temporal history measurements with spectral resolution

Although its importance is sometimes underestimated, a compact small design is vital in scientific experiments that are often carried out in relatively small target vacuum chambers. Moreover, for spectroscopic applications it is often

* kuba@1lnl.gov; phone +1 (925) 4244390 
required that the streak camera slit be positioned on the focal axis of an imaging crystal. For a large camera that would mount to a chamber wall, this would mean an intrusive re-entrant tube, blocking much of the target solid angle.

In this paper, we describe the sub-picosecond LLNL T-REX streak camera. This camera was the first of several streak cameras that eventually broke 1-ps frontier. The streak camera has since been successfully used in several scientific applications that demonstrate the streak camera's unique capabilities. For example, the relatively small dimensions of the camera combined with a von Hámos spectrometer enabled high-resolution spectroscopic studies of short-pulse laser.

In the following section we will give a brief overview of the streak camera design and its characteristics. In section 3 , examples of scientific applications of the T-REX streak camera in a wide range of wavelengths are reported: (a) infrared, visible and UV laser pulse measurements, (b) temporal history study of a transient soft-x-ray laser at $14.7 \mathrm{~nm}$ $(84 \mathrm{eV})$, and (c) an example of applications in hard x-rays at $\sim 1-2 \mathrm{keV}$, where a spectroscopic study of heated thin foils with a relativistic-intensity short-pulse-laser was carried out.

\section{T-REX STREAK CAMERA DESIGN}

\section{General Considerations}

The temporal resolution of a streak camera is limited by a quadratic sum of the resolution losses throughout the streak camera [2]. In other words, supposing Gaussian shapes of the respective de-convolution functions, one can show that

$$
\delta \tau_{R}=\sqrt{\left(\delta \tau_{1}\right)^{2}+\left(\delta \tau_{2}\right)^{2}+\ldots},
$$

where $\delta \tau_{R}, \delta \tau_{1}$, and $\delta \tau_{2}$ are the total temporal resolution and the FWHM duration of the response deconvolution functions of the resolution losses on each element, respectively. In general, the x-ray streak camera resolution limitations can be separated into three physical effects:

- Temporal dispersion of the electron bunch

- Space-charge spreading of the electron bunch, and

- Poor spatial resolution when detecting the electron bunch

In the previous generation of streak cameras with the resolution on the order of $2 \mathrm{ps}$, the latter two effects rarely play a role, but they need to be taken into account in the new sub-ps streak camera.

Most losses in resolution can be ascribed to the temporal dispersion of an electron bunch when it is accelerated between the photocathode and the accelerating mesh (and right after passing through it). The bulk of this dispersive loss is proportional to [2]

$$
\delta \tau \propto \frac{\left(E_{0}+\delta E_{0 S} / 2\right)^{1 / 2}-\left(E_{0}-\delta E_{0 S} / 2\right)^{1 / 2}}{E_{\text {field }}}
$$

where $E_{0}$ is the kinetic energy of the electrons, $\delta \mathrm{E}_{0 \mathrm{~S}}$ is the FWHM energy spread of the electron energy distribution, and $E_{\text {field }}$ is the electric field strength of the accelerating field.

The energy of the released electrons from the photocathode, $\mathrm{E}_{0}$, remains relatively small (1-3 eV), however one can maximize the temporal resolution by (1) optimizing the energy spread, $\delta \mathrm{E}_{0 \mathrm{~S}}$, (that has to be as small as possible) and (2) maximizing the electric field accelerating the electrons, $E_{\text {field }}$. In most streak cameras CsI photocathodes are used because of their sensitivity. The energy spread could be reduced by choosing a different photocathode material, but the small improvement would come at the expense of sensitivity. The main improvement in temporal resolution can therefore be achieved by maximizing the accelerating field and minimizing the path on which the electrons are 
accelerated. Additionally, the photocathode's surface quality can help to minimize the electron energy spread.

\section{Mesh and photocathode}

Both the direction and energy of the electrons immediately off the photocathode are associated with the surface distortions of the photocathode. In order to minimize surface distortions a special procedure has been developed: $250 \AA$ of $\mathrm{Si}_{3} \mathrm{Ni}_{4}$ is coated on a $1.25 \mathrm{~cm}$ x $4.00 \mathrm{~cm}$ x $300 \mu \mathrm{m}$ silicon wafer. Subsequently, the silicon is etched away from a 100$\mu \mathrm{m}$ wide, $2.54-\mathrm{cm}$ long area, producing an optical quality window that will act as the streak camera's aperture slit. On the unetched side, the window is coated with $400 \AA$ of $\mathrm{Au}$ (or, alternatively, $\mathrm{Al}$ for a certain range of wavelengths) to provide electrical conductance. Finally, an $850-\AA$ thick layer of CsI is coated on top of the Au. The design is relatively simple and the photocathodes can be readily replaced in case damage occurs. The photocathode is mounted separately from the photocathode grid, which further simplifies manipulation and possible replacement of a photocathode. The photocathode is pulse-charged from an external circuit to a negative voltage of $-15 \mathrm{kV}$ with the pulse length of $\sim 5 \mathrm{~ns}$. The relatively long pulse was chosen arbitrarily in order to simplify streak camera's timing and it guarantees a constant voltage while the electrons are being produced. The accelerating 394-wires/cm (1000-wires/inch) grid is placed at 500 $\mu \mathrm{m}$ from the photocathode. For the sake of a simple design, the grid is held at ground (zero) voltage, while the negative voltage at the photocathode provides an accelerating electric field of $\sim 300 \mathrm{kV} / \mathrm{m}$ over the width of $500 \mu \mathrm{m}$ between the photocathode and the mesh.

\section{Electron Optics}

After being accelerated the electron bunches are relayed to the streak camera's electron optics. The optics consists of two electro-static "cylindrical" lenses perpendicular to each other. The "temporal" lens focuses the electron beam in the direction perpendicular to the streak camera's slit (the direction of the temporal resolution at the output of the streak camera), while it does not affect the beam in the perpendicular (so-called "spatial") direction. The temporal lens is used to image the aperture slit at the output phosphor. Its proper design and alignment is hence essential for the resolution of the streak camera. The "spatial" lens (affecting the beam in the direction along the slit) is designed to focus the electrons from the length of the slit and hence operates at a negative high voltage. The two lenses are isolated from each other by a 180 -wires/cm (300-wires/inch) mesh at zero potential. The optimization of both lenses is important not only for an achievement of the highest possible resolution, but also in order to reduce the streak camera size and therefore enable a compact design. The streak camera's electron optics system has been, therefore, carefully modeled by the SIMION 3D 7.0 ion optics code.

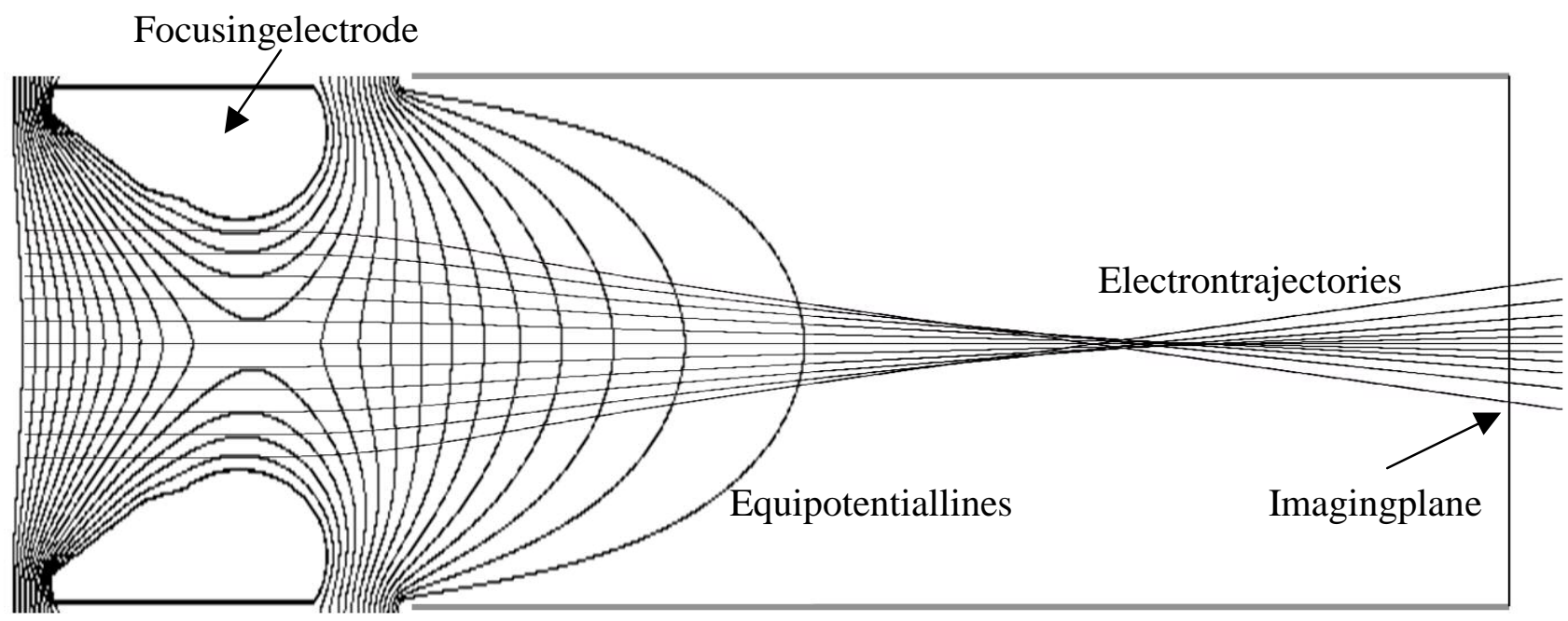

Figure 1: Specific "lobe" shape of electrodes and etched equipotential lines reduce significantly the size of the streak camera's spatial lens. The lens' compact design has been developed through electron optics modeling by the SIMION numerical code. Electron beams are supposed to be parallel at the input zero potential mesh and are imaged onto a phosphor by the focusing electrodes. 
The code calculates electron trajectories in electric (magnetic) fields and equipotential lines of the fields. The program solves the Laplace equation with electrodes acting as boundary conditions. Given a defined simulation volume, the program determines trajectories of injected electrons from the calculated electrostatic potential distribution. Current simulations of each lens have assumed a two-dimensional environment; in other words all electrodes are taken to be infinitely long. Since the electrons produced by the photocathode are accelerated by the potential difference of $15 \mathrm{kV}$ between the mesh and the photocathode, electrons are injected into simulations with an initial energy of $15 \mathrm{keV}$. The SIMION code does not support Poisson solutions; space-charge effects are estimated from charge repulsion methods. One such method considers electrons as line charges and computes their charge repulsion from each other. This approach is effective in predicting when space-charge effects become significant, however, it shows a lower accuracy when predicting the resultant electron trajectories.

The original simulations of the spatial lens were run with standard rod electrodes forming an "ideal" electron lens; the SIMION code helped to optimize their positions and voltages. The resulting equipotential lines were then used to simplify the design of the streak camera's lens (Fig. 1). In the actual design, the electrode shape was modified to reproduce an equipotential line as predicted by the SIMION code. The new "lobe" shaped electrodes then reduce significantly the size of the spatial lens and hence the whole streak camera, allowing for the use of a substantially lower voltage that would otherwise be needed, and, at the same time, retain the optimum electric field structure. Moreover, in order to simulate infinitely long electrodes (equivalent to a 2-D calculation), the shapes of the equipotential lines are etched into a printed circuit board and connected to a voltage divider.

In order to provide a temporal resolution of the electron bunch, the photoelectrons are swept using parallel sweeping plates. The sweep plates are oriented so that the field between the plates is perpendicular to the length of the photocathode slit. Each plate is charged with a 250 -ps risetime, $4-\mathrm{kV}$ voltage pulse of opposite polarity through a split coaxial network transmission line. The voltage risetime on the sweep plates is $200 \mathrm{ps}$ through the transmission line. The field uniformity was determined to be $1-2 \%$ over the $2.5-\mathrm{cm}$ length of the sweep plates. The electron sweeping was found to introduce a negative lens effect in the temporal direction, increasing the temporal response. To offset the effect, the temporal lens voltage was being increased until the minimum duration time history was found at the output from the streak camera detected by a phosphoresque screen. The swept photoelectrons strike a fiber optic coated with P-20 phosphor. The phosphor coated fiber optic is interfaced to a fiber optic, micro-channel plate image intensifier. The image is read out by coupling a $1024 \times 1024$ CCD with a fiber optic 1:2 face-plate to the image intensifier. The entire streak camera and read-out system is $50 \mathrm{~cm} \times 7.6 \mathrm{~cm}$ x $7.6 \mathrm{~cm}$, making it small enough for many vacuum chambers used in laser-plasma interaction experiments.

These substantial design improvements over the previous generation of streak cameras result in the excellent streak camera parameters: the T-REX streak camera can routinely be operated at $\sim 500$-fs resolution (with a dynamic range of $\geq$ 10), and for certain combinations of the incident light wavelength and a photocathode a resolution of up to $\sim 300 \mathrm{fs}$ (however with a minimal dynamic range) has been demonstrated. Its relatively small size allows the streak camera to be incased into a $50-\mathrm{cm} \times 10-\mathrm{cm} \times 10-\mathrm{cm}$ (including shielding) volume. Additionally, a new unique photocathode-mesh design reduces damage when electrical breakdown occurs between the photocathode and the mesh.

\section{STREAK CAMERA CHARACTERIZATION AND APPLICATION IN EXPERIMENTS}

\section{STREAK CAMERA RESOLUTION - UV, VISIBLE AND IR LASER PULSES}

The T-REX streak camera was characterized using a Michelson interferometer and short (50 fs) laser pulses in the UV, visible and infrared wavelength range. The interferometer splits the incident laser beam and allows fine timing of the both arms to achieve exact delays between them. "Zero" delay - the same length for both interferometer arms - is reached when interference fringes occur. They can be observed whether at a test screen or directly at the streak camera in 
DC (focus) mode, in which the sweeping plates are switched off and the electron optics images the streak camera's entrance slit.

The interferometer in this set-up enables one to evaluate the streak camera resolution using two main habitual criteria: (1) Rayleigh criterion and (2) Convolution criterion. The Rayleigh criterion defines the resolution as the minimal delay between two incident pulses that can still be distinguished by the streak camera as being clearly separate. In Fig. 2, the delay between two pulses was set by the Michelson interferometer at 1 ps and several laser shots were recorded on a CCD. The pulses can still be clearly distinguished, which shows a sub-picosecond resolution of the streak camera. The convolution criterion assumes a delta function (or a very short laser pulse compared to the expected resolution) incident at the aperture slit of the streak camera. The delta function is then imaged by the streak camera at its detection system as a finite temporal history pulse. In other words, the delta function is convolved with the streak camera's resolution function. The resolution is then defined by the FWHM of the recorded pulse. The convolution function can be used in deconvolving very short measurements close to the resolution limit to obtain the real duration of a measured pulse. The lineout of the data taken with the Michelson interferometer (Fig. 2b) enables one to determine the resolution of the streak camera to be $\sim 500 \mathrm{fs}$.
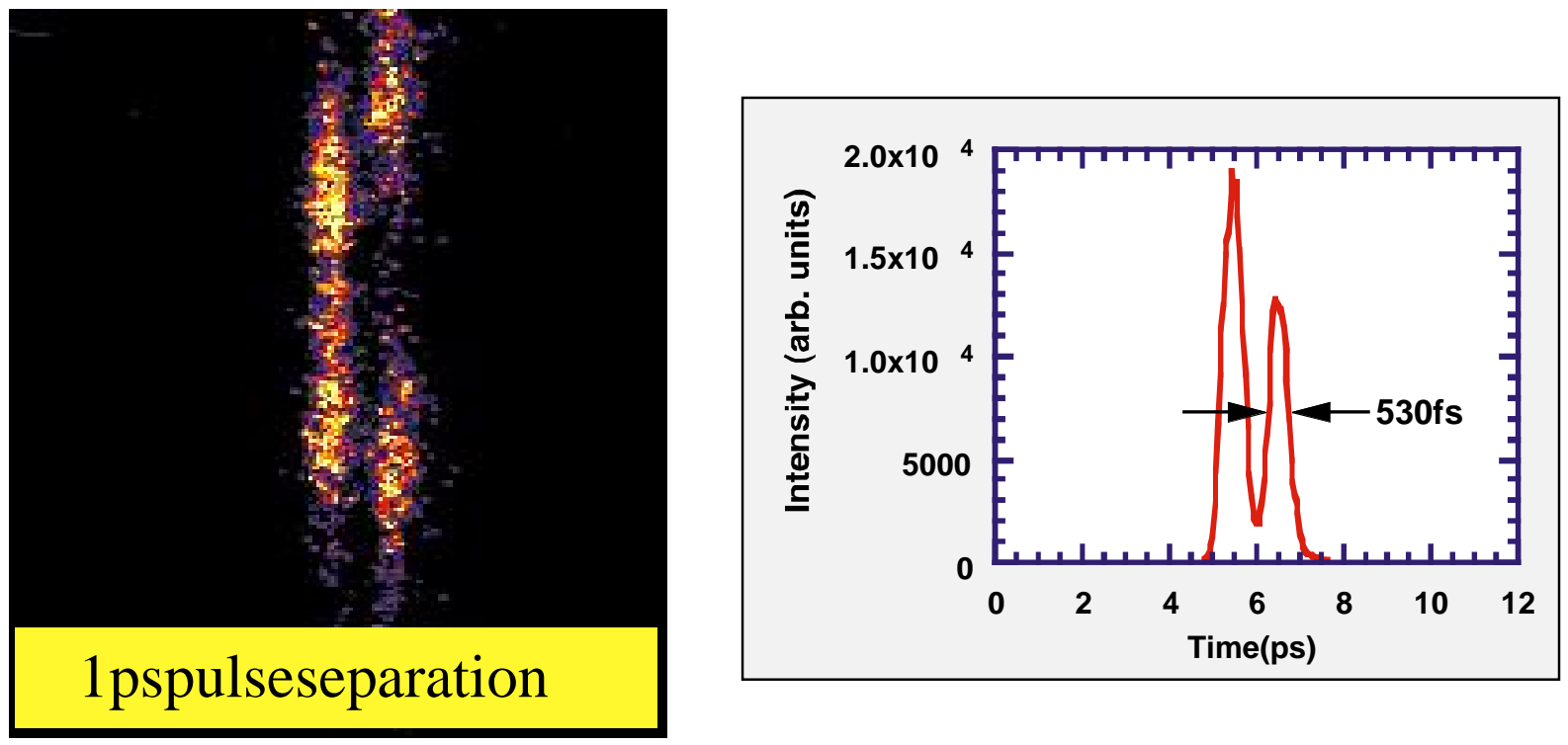

Figure 2: The resolution of the streak camera is well bellow 1 ps. An optical 50-fs long laser pulse was split and the two resulting pulses were then mutually delayed by 1 ps. They can be clearly distinguished at the streak camera output as recorded by a CCD. The line-out ( $2 b$ ) shows a resolution of $\sim 500$ fs.

\section{TEMPORAL CHARACTERIZATION OF A TRANSIENT X-RAY LASER AT 14.7 NM}

Since their first clear demonstration by Matthews et al. (LLNL) in 1985 [3], significant progress has been made in improving the efficiency and characteristics of X-ray lasers (XRL's). Various types currently available, including different schemes using a laser or capillary discharge as a pump, enable a wide range of x-ray laser pulse parameters suitable for a variety of scientific applications. In particular, a determined effort has been undertaken to develop a reliable saturated "table-top" XRL operating at short wavelengths with short pulse durations. The device would be a versatile tool for research into fast processes, such as those in petawatt laser plasmas or inertial confinement fusion plasma research. 
A promising transient scheme consists in a two-stage target irradiation: The initial 300-600-ps low-intensity pulse creates the plasma with a large abundance of the desired ion species (i.e., nickel-like, neon-like or possibly others). The second, high intensity (sub)picosecond, laser pulse heats the preformed plasma and, by collisions between free electrons and ions, creates a transient population inversion. Since the population inversion is short lived $(<10 \mathrm{ps})$ compared with the photon transit time in the active medium along the target ( $\sim 33 \mathrm{ps}$ for a 1-cm target), the ultrashort laser pulse must heat the plasma only locally and - by the guillotine effect - make the gain region "travel" at the velocity of the XRL photons being amplified. This is called traveling-wave (TW) irradiation geometry. The beneficial effect of the TW was observed to increase the XRL output intensity by a factor of 300-400, compared with the non-TW irradiation for a 400-fs
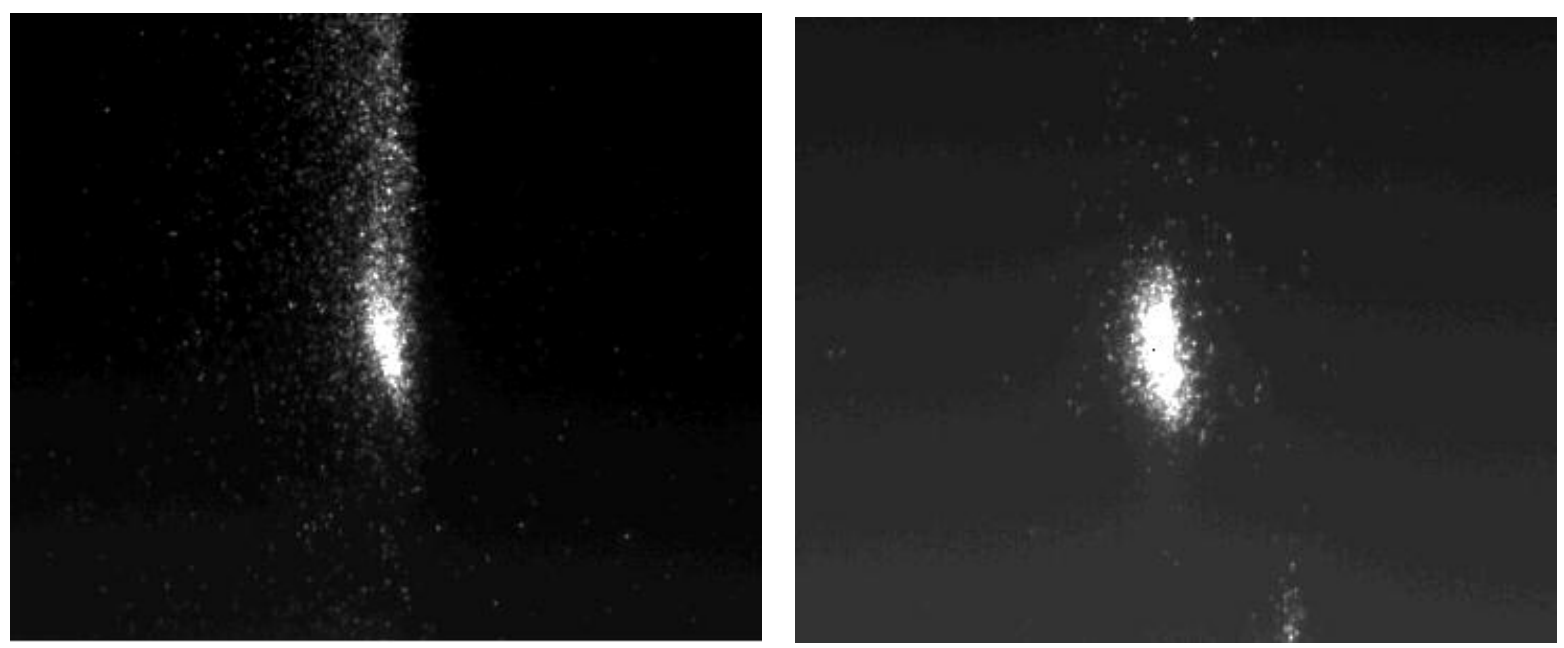

Figure 3: The x-ray laser pulse duration varies from $\sim 2.6-4$ ps when the short pump pulse duration changes from 600 fs to 5 ps. The soft $\mathrm{X}$-ray laser signal at $14.7 \mathrm{~nm}$ with a temporal and spatial resolution in the horizontal direction as observed by the T-REX streak camera 
heating pulse [4]. With the TW on, a gain of $33 \mathrm{~cm}^{-1}$ with a saturation length of $3.8 \mathrm{~mm}$ was observed during this experiment.

The combination of a short duration high gain and the TW irradiation geometry results in $~ 2$-ps XRL pulses for the Nilike $4 \mathrm{~d}-4 \mathrm{p}$ silver transition at $13.9 \mathrm{~nm}$, which are the shortest XRL pulses observed up to now [5]. Preliminary results on the XRL pulse duration in dependence on pump laser parameters have been discussed in [4]. Theoretical studies of these experiments showed that the gain duration in transient plasmas is cut-off by over-ionization [6], [7] suggesting that adjusting pump parameters, especially the duration of the short pump pulse, may result in a tunable XRL pulse length.

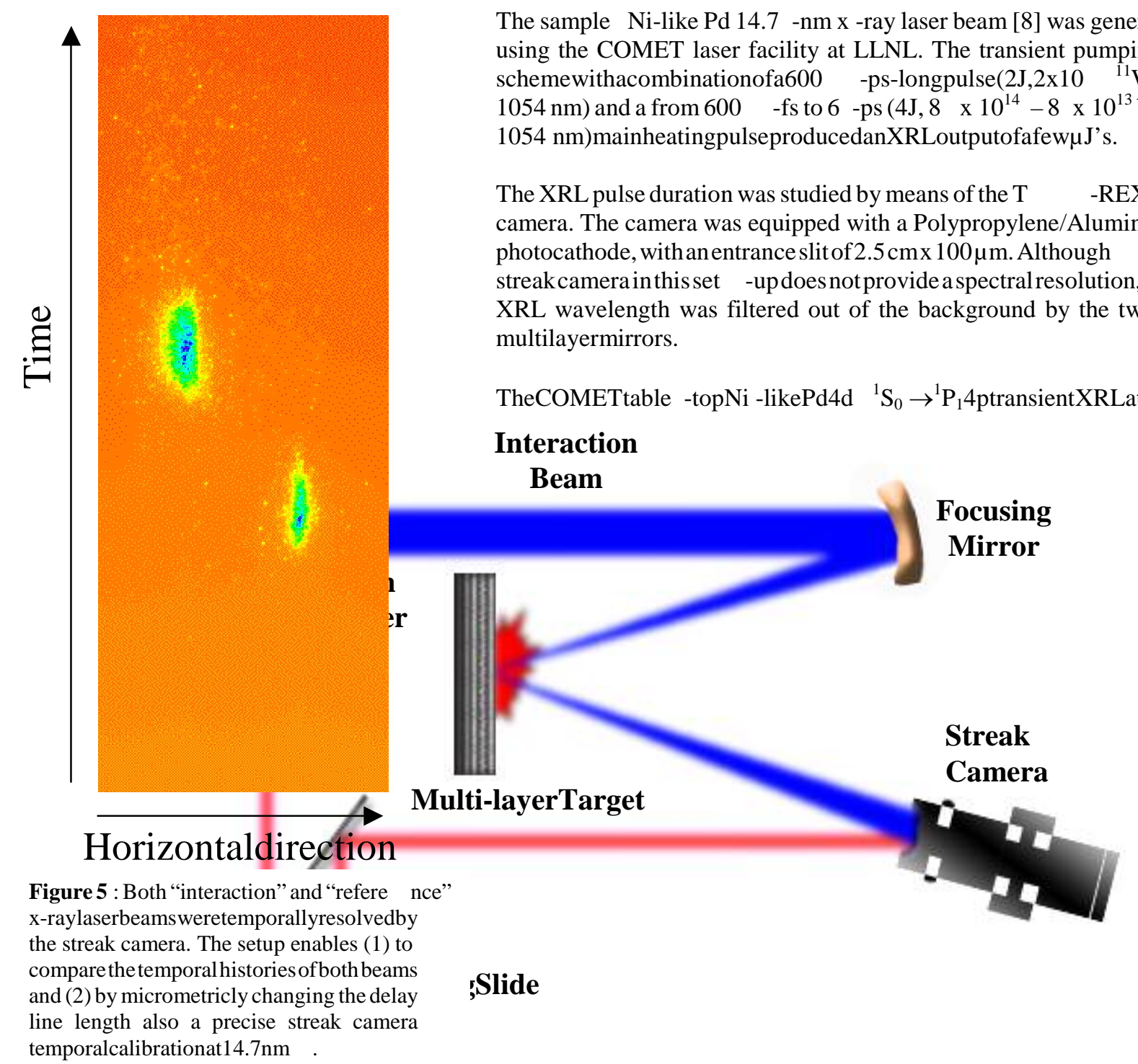

Figure 4: Experimental setup for the temporally resolved damage experiment.

$\mathrm{nm}$ has been optimized during several previous experimental runs in order to maximize the XRL output [8]. In this sense, the "optimum" conditions were found for a short pulse of 5-6 ps at FWHM, with maximum available pump 
energy of $4 \mathrm{~J}$, while the long 600-ps pulse typically runs between 2 and $3 \mathrm{~J}$ on target. The peak-to-peak delay was optimized for $700 \mathrm{ps}$.

The temporal history of an XRL pulse under these "optimal" pumping conditions (Fig. 3) with a relatively long heating pulse was measured to be $(4.0 \pm 0.5) \mathrm{ps}$, which is relatively long, compared to 2 ps observed in previous experiments [5]. With the aim to shorten and control reliably the x-ray laser pulse duration within the range suggested, we studied the pulse histories in dependence on heating pump laser pulse duration. We obtained several data points for three different pulse durations: from the shortest available at COMET - $600 \mathrm{fs}$ - up to the "optimum" pump conditions with heating laser pulse length of $6 \mathrm{ps}$. The shortest pulse we were able to demonstrate achieved $2.6 \pm 0.5 \mathrm{ps}$, which is comparable to the pulses demonstrated so far [5].

Contrary to this previous experiment, the current COMET experimental campaign provided both temporal and spatial resolution of the x-ray laser pulses at the expense, however, of the spectral resolution. The lack of the spectral resolution makes it difficult to verify the relative temporal position of the x-ray laser pulse and the background Bremsstrahlung radiation measured in the previous work as it is impossible to separate both radiative effects. The previously observed occurrence of the x-ray laser pulse before the Bremsstrahlung peak was explained by numerical simulations as being predetermined by relatively short-lived gain that appears before it is cut-off in the overionization region. The interconnection between the overionization and the gain would suggest that the shorter the heating pulse (that is kept at the same energy) the shorter is the resulting x-ray laser pulse. Therefore, the present streak camera measurements support the previously shown relations in x-ray laser Ni-like plasma. Further calculations simulating the gain and ionization abundances in dependence on the heating laser pulse energy are however needed.

The spatial resolution combined with the temporal resolution of the streak camera provides new insights into the $\mathrm{x}$-ray laser plasma dynamics. Interesting effects in the spatio-temporal development were observed for shorter heating laser pulses of $600 \mathrm{fs}$ and $1.2 \mathrm{ps}$. The x-ray laser output is observed to move with time with respect to the target, which could suggest that the highest gain region moves with the expanding plasma. Further modeling that is beyond the scope of these proceedings on the T-REX streak camera is currently being carried out.

The following part of the experiment concentrated on focusing the x-ray laser beam in order to study an interaction of the focused x-ray laser beam with multi-layer targets. The temporal history of the pulse reflected from the sample is compared on a shot-to-shot basis with the original pulse, a small portion of which is sent directly to the streak camera without the focusing and interaction with the target (Fig. 4).

Although the achieved focal size did not allow us to observe damage on a sample, we were able to validate the method and compare both the "interaction" and "reference" beam at the streak camera (Fig. 5). This experimental setup also enables an accurate streak camera calibration at this wavelength in terms of pulse duration by adjusting micrometrically the timing slide at different known positions. 


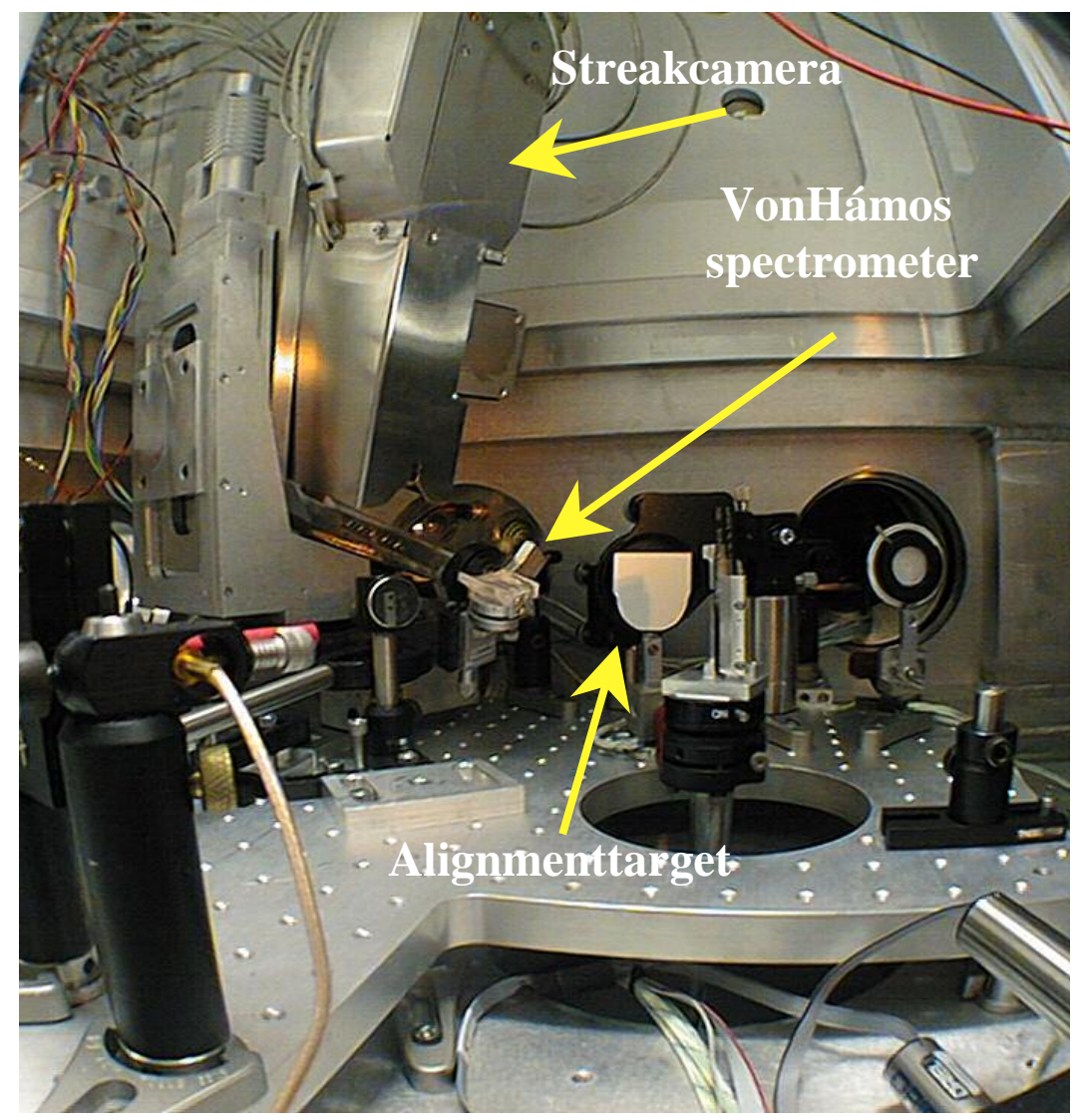

Figure 6: The streak camera setup with the von Hámos spectrometer. The compact design of the streak camera makes possible various complicated experimental geometries to be realized such as those for high-resolution spectral measurements. The von Hámos spectrometer is placed at $8 \mathrm{~cm}$ from the target and the $\mathrm{x}$-rays produced at target are spectrally resolved and imaged onto the streak camera aperture slit. The setup therefore provides temporal resolution of the generated spectra. 


\section{APPLICATION AT 1-2 KEV: HEATING OF THIN FOILS WITH A RELATIVISTIC-INTENSITY SHORT-PULSE LASER}

As a matter of example we will mention a spectroscopic experiment that was recently carried out with the T-REX streak camera operating in 1-2-keV x-ray region [9]. The experiment concentrated on K-shell x-ray spectroscopy of sub$100 \mathrm{~nm}$ Al foils irradiated by high contrast, $150-\mathrm{fs}, 150-200-\mu \mathrm{J}$ laser pulses with the focused intensity of $\sim 10^{19} \mathrm{~W} / \mathrm{cm}^{2}$. The resulting $\mathrm{x}$-rays were studied by the T-REX streak camera with 500-fs resolution coupled to a dual-crystal von Hámos spectrometer. The von Hámos crystals focus the x-ray spectra along the streak camera aperture slit. The streak camera then provides the temporal resolution of the emitted x-ray spectra. Two RbAP crystals [Rubidium phtalate or $\mathrm{C}_{6} \mathrm{H}_{4}(\mathrm{COOH})(\mathrm{COORb})$, with $2 \mathrm{~d}$ spacing of $\left.2.590 \mathrm{~nm}\right]$ were bent to 3.6 and $3.0 \mathrm{~cm}$ radii to provide - in a single shot two different spectral regions (specifically in this experiment, the spectral region around $1 \mathrm{~s}^{2}-1 \mathrm{~s} 2 \mathrm{p} \mathrm{He}$ transition and $1 \mathrm{~s}^{2}-$ $1 \mathrm{~s} 3 \mathrm{p} \mathrm{He}_{\beta}$ transition). It is worth noting that the distances target-spectrometer and spectrometer-streak camera and the angle of the focusing plane are fixed by crystals' radii and $2 \mathrm{~d}$ crystal spacing. It is therefore important that the streak camera be relatively small and compact as it is often operated in unusual geometries close to the target (Fig. 6).

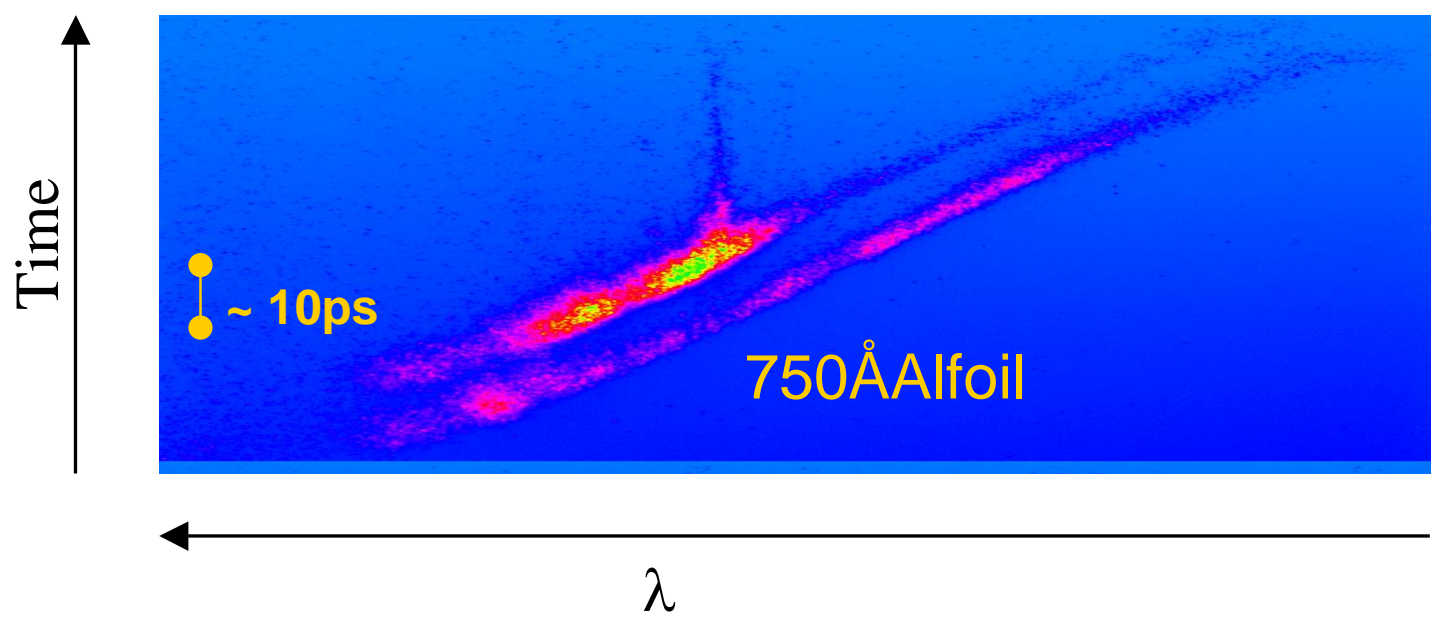

Figure 7: Time resolved spectra of the laser heated $\mathrm{Al}$ foil as taken by the T-REX streak camera

An example of the measured spectra is shown in Fig. 7. The transit time difference between different wavelengths (reflected off the two different crystals) results in an image of two spectra shifted in time with respect to each other. Further, the transit time difference between different wavelengths reflected off a single crystal was used to calibrate and check the linearity of the sweep for each shot. Spectra from both crystals are inclined because of the time delay between low and high energy photons when observed by a streak camera under grazing incidence; in other words, it results from path difference between each part of the photocathode as given by the setup geometry.

\section{CONCLUSIONS AND PERSPECTIVES}

A sub-picosecond streak camera has been developed at the Lawrence Livermore National Laboratory and it was successfully applied in a range of scientific experiments. The T-REX streak camera routinely operates at $\sim 500$ fs with a relatively large dynamic range $(\geq 10)$; the resolution down to $\sim 300 \mathrm{fs}$ (with a small dynamic range) has been however already demonstrated. The compact design $(50 \mathrm{~cm} \times 10 \mathrm{~cm} \times 10 \mathrm{~cm})$ of this streak camera makes it a very suitable device for an operation in plasma physics experiments that are often carried out in unusual geometries and limited-space 
target chambers. Moreover, it also enables coupling the streak camera with a von Hámos spectrometer, and the set-up provides both temporal and spectral resolution of the emitted radiation.

The streak camera temporal resolution has been characterized both by Rayleigh and convolution criteria with IR, visible and UV short laser pulses. A successful application in a transient x-ray laser research at $14.7 \mathrm{~nm}$ enabled us to demonstrate $\mathrm{x}$-ray pulses as short as 2-6 ps, the duration of which can be controlled by pump laser parameters. Demonstration of the spatial x-ray laser pulse distribution evolving in time will bring important insight into x-ray laser dynamics. Finally, the streak camera is being routinely applied in various spectroscopic hard x-ray experiments at 1-2 $\mathrm{keV}$. The experimental setup takes advantage of the streak camera's small dimensions that enable its coupling with a von Hámos spectrometer.

The streak camera proved to be a useful tool in plasma physics. Its further development is aimed not only at the temporal resolution improvements, but also at an extension of its applicability to other domains, such as hard x-ray studies at the PLEIADES source [10] that produces x-ray pulses between 10-100 keV.

\section{REFERENCES}

1. M. M. Murnane, H. C. Kapteyn, and R. W. Falcone: X-ray streak camera with 2 ps response, Appl. Phys. Lett. 56, 1948 (1990).

2. D. J. Bradley, A. G. Roddie and W. Sibbett, M. H. Key, M. J. Lamb, C. L. S. Lewis and P. Sachsenmaier: Picosecond x-ray chronoscopy, Opt. Comms 15, 231 (1975).

3. D. L. Matthews, P. L. Hagelstein, M. D. Rosen, M. J. Eckart, N. M. Ceglio, A. U. Hazi, H. Medecki, B. J. MacGowan, J. E. Trebes, B. L. Whitten, E. M. Campbell, C. W. Hatcher, A. M. Hawryluk, R. L. Kauffman, L. D. Pleasance, G. Rambach, J. H. Scofield, G. Stone, and T. A. Weaver, Phys. Rev. Lett. 54, 110-113 (1985).

4. A. Klisnick, J. Kuba, D. Ros, R. Smith, G. Jamelot, C. Chenais-Popovics, R. Keenan, S. J. Topping, C. L. S. Lewis, F. Strati, G. J. Tallents, D. Neely, R. Clarke, J. Collier, A. G. MacPhee, F. Bortolotto, P. V. Nickles, K. A. Janulewicz: Demonstration of a 2-ps transient x-ray laser, Phys. Rev.A 65,033810 (2002).

5. J. Kuba, A. Klisnick, D. Ros, P. Fourcade, G. Jamelot, J.-L. Miquel, N. Blanchot, J.-F. Wyart: Two color transient pumping in Ni-like silver at 13.9 and $16.1 \mathrm{~nm}$, Phys. Rev. A 62, 043808 (2000).

6. J. Nilsen, and J. Dunn, "Modeling of short-pulse-driven nickel-like X-ray lasers and recent experiments", E. E. Fill, J. J. Rocca eds., Proc. of the SPIE Conf. 4505, 100-106 (2001).

7. Jaroslav Kuba, Raymond F. Smith, Djamel Benredjem, Clary Möller, Lee Upcraft, Robert King, Annie Klisnick, Ladislav Drska, Geoff J. Pert, Jean-Claude Gauthier: Modelling of the transient Ni-like Ag X-ray Laser, J. Opt. Soc. Am. B 20, 208 (2003).

8. J. Dunn, Y. Li, A. L. Osterheld, J. Nilsen, J. R. Hunter, and V. N. Shlyaptsev: Gain saturation regime for laserdriven tabletop, transient Ni-like ion X-ray laser, Phys. Rev. Lett. 84, 4834 (2000).

9. P. Audebert, R. Shepherd, K. B. Fournier, O. Peyrusse, D. Price, R. Lee, P. Springer, J.-C. Gauthier, and L. Klein: Heating of thin foils with a relativistic-intensity short-pulse laser, Phys. Rev. Lett. 89, 265001 (2002).

10. Jaroslav Kuba, Scott G. Anderson, C. P. J. Barty, Shawn M. Betts, Rex Booth, Winthrop J. Brown, John K. Crane, Robert R. Cross, David N. Fittinghoff, David J. Gibson, Fred V. Hartemann, Gregory P. Le Sage, James B. Rosenzweig, Aaron M. Tremaine, and Paul T. Springer: PLEIADES: High Peak Brightness, Subpicosecond Thomson Hard-X-ray Source, Soft X-ray Lasers and Applications V (AM304), Section Hard X-Rays and FELs, Ernst E. Fill and Szymon Suckewer, Conference chairs, SPIE International Symposium on Optical Science and Technology, San Diego, California, USA, 3 - 8 August 2003.

\section{ACKNOWLEDMENT}

Work performed under the auspices of the US Department of Energy by the University of California Lawrence Livermore National Laboratory under Contract No. W-7405-Eng-48. 